\title{
NEW OBSERVATIONS OF LBV ENVIRONMENTS
}

\author{
NOLAN R. WALBORN ${ }^{1}$ and IAN N. EVANS \\ Space Telescope Science Institute ${ }^{2}$ \\ 3700 San Martin Drive \\ Baltimore, Maryland 21218, USA \\ EDWARD L. FITZPATRICK ${ }^{1}$ \\ Princeton University Observatory \\ Peyton Hall \\ Princeton, New Jersey 08544, USA \\ MARK M. PHILLIPS \\ Cerro Tololo Inter-American Observatory ${ }^{3}$ \\ Casilla 603 \\ La Serena, Chile
}

\begin{abstract}
Two observational programs which provide new information about particular LBVs through investigations of their immediate surroundings are described. (1) Digital spectral classification of $\mathrm{OB}$ supergiants in compact groups apparently associated with Radcliffe 127 and S Doradus has revealed several interesting objects and indicates which of them are likely to be generically related to the LBVs. (2) Velocity-resolved images of the Eta Carinae shell show qualitatively new features, which will contribute substantially to the interpretation of its complex spatial/kinematical structure.
\end{abstract}

\section{OB Supergiants Associated with Radcliffe 127 and S Doradus}

Normal OB stars associated with massive peculiar objects may provide indirectly, valuable information about the physical nature of the latter, such as the initial mass, age, and evolutionary status, although even in the Large Magellanic Cloud uncertainties with regard to coeval formation and chance alignments may arise. Several Luminous Blue Variables (Davidson, Moffat, and Lamers 1989) and Ofpe/WN9 objects (Bohannan and Walborn

1 Visiting Astronomer, Cerro Tololo Inter-American Observatory, ${ }^{3}$ National Optical Astronomy Observatories, operated by the Association of Universities for Research in Astronomy, Inc., under contract with the National Science Foundation.

2 Operated by the Association of Universities for Research in Astronomy, Inc., under contract with the National Aeronautics and Space Administration. 
1989 ) in the LMC are apparently located in compact OB groups or multiple systems (Walborn 1989), only one of which has been investigated in detail to date (Schild 1987). Two further outstanding examples are provided by the currently active LBVs R127 = HDE $269858=$ Sanduleak (Sk) $-69^{\circ} 220$ (NGC 2055, Figure 1) and S Dor $=$ R88 $=$ HD 35343 $=\mathrm{Sk}-69^{\circ} 94$ (Fig. 2 of Leitherer et al. 1985). NRW and ELF have obtained blue-violet digital classification spectrograms of four $\mathrm{OB}$ supergiants in the former group and one in the latter, with the two-dimensional photon-counting system at the CTIO $1 \mathrm{~m}$ telescope during (with one exception) December 1989. These data have a resolution of $1.5 \AA$ and $\mathrm{S} / \mathrm{N}$ per resolution element of about 80 ; they were obtained and processed identically to those in the extensive OB digital atlas of Walborn and Fitzpatrick (1990), with respect to which they have been classified.

Four of these spectrograms are reproduced in Figure 2 in the WF Atlas format; the fifth, of R128 = HDE $269859=\mathrm{Sk}-69^{\circ} 221$, will be included in the extensive discussion of LMC B-supergiant spectra by Fitzpatrick (1990). These spectra are of considerable interest in their own right: all of them display emission lines, CNO anomalies, and/or variability. $\mathrm{Sk}-69^{\circ} 217$ is a previously unknown LMC Of supergiant, with broad emission underlying the narrow N III $\lambda \lambda 4634,4640-42$ and He II $\lambda 4686$ emission lines. Sk $-69^{\circ} 218$ and 226 are new OBN supergiants: note the extreme weakness of C III $\lambda \lambda 4070,4650$. The former spectrum shows weak N III $\lambda 4640$ emission, while the latter is definitely variable and warrants further investigation: half of the four summed individual observations were obtained on each of two consecutive nights, between which substantial changes in the intensities of several He I, Si IV, N II, and N III lines occurred. In striking contrast with the previous two spectra, that of $S$ Dor No. 1 is nitrogen deficient: note the weakness of $\mathrm{N}$ III $\lambda 4097$ in the blue wing of $\mathrm{H} \delta$, in comparison with the other two. Finally, Sk-69 221 (not shown here), also has a nitrogen-deficient spectrum.

The spectral classifications of these stars are given in Table 1, along with other observed and derived parameters as follows: $M_{V}$ from the spectral-type calibrations of Walborn (1972, 1973); $V$ and $B-V$ from Ardeberg et al. (1972) or Isserstedt (1975), and $E_{B-V}$ based on the intrinsic colors of Johnson (1966-Fitzpatrick 1988 has derived somewhat redder values for $\mathrm{LMC} O \mathrm{OB}$ supergiants, but the differences are not significant for the present purpose), except for $S$ Dor No. 1 whose values are derived from a photographic B magnitude estimate and the $E_{B-V}$ of S Dor quoted by Leitherer et al. (1985); $M_{V}$ derived from the photometry with $R=3$ and a distance modulus of 18.6 for the LMC; $M_{b o l}$ corresponding to the latter $M_{V}$, and $\log T_{e f f}$, both from the calibration of Humphreys and McElroy (1984); and finally, estimates of the initial stellar masses determined by plotting the preceding two parameters on the theoretical HR diagram for $Z=0.005$ by Maeder (1990), as further discussed below.

The large discrepancies between the spectroscopic and photometric $M_{V}$ 's for three of the late-O/early-B supergiants in Table 1 , whose luminosity classes are based on $\mathrm{Si} / \mathrm{He}$ line ratios relative to galactic standards, but not for the Of object whose luminosity class depends on the He II emission, are identical to the situation found by Walborn (1977), and may well be due to the LMC metal deficiency, similar to but less extreme than the SMC case (Walborn 1983). The definition of primary classification standards in the Magellanic Clouds is an important problem for future work. Curiously, there is no significant discrepancy in the case of S Dor No. 1, which could indicate a range of $\mathrm{Si}$ abundances in the LMC, as was suggested in the SMC (Walborn 1983), but note that the photometry of this star is uncertain. The photometric $M_{V}$ 's have been used for the $M_{b o l}$ determinations. 


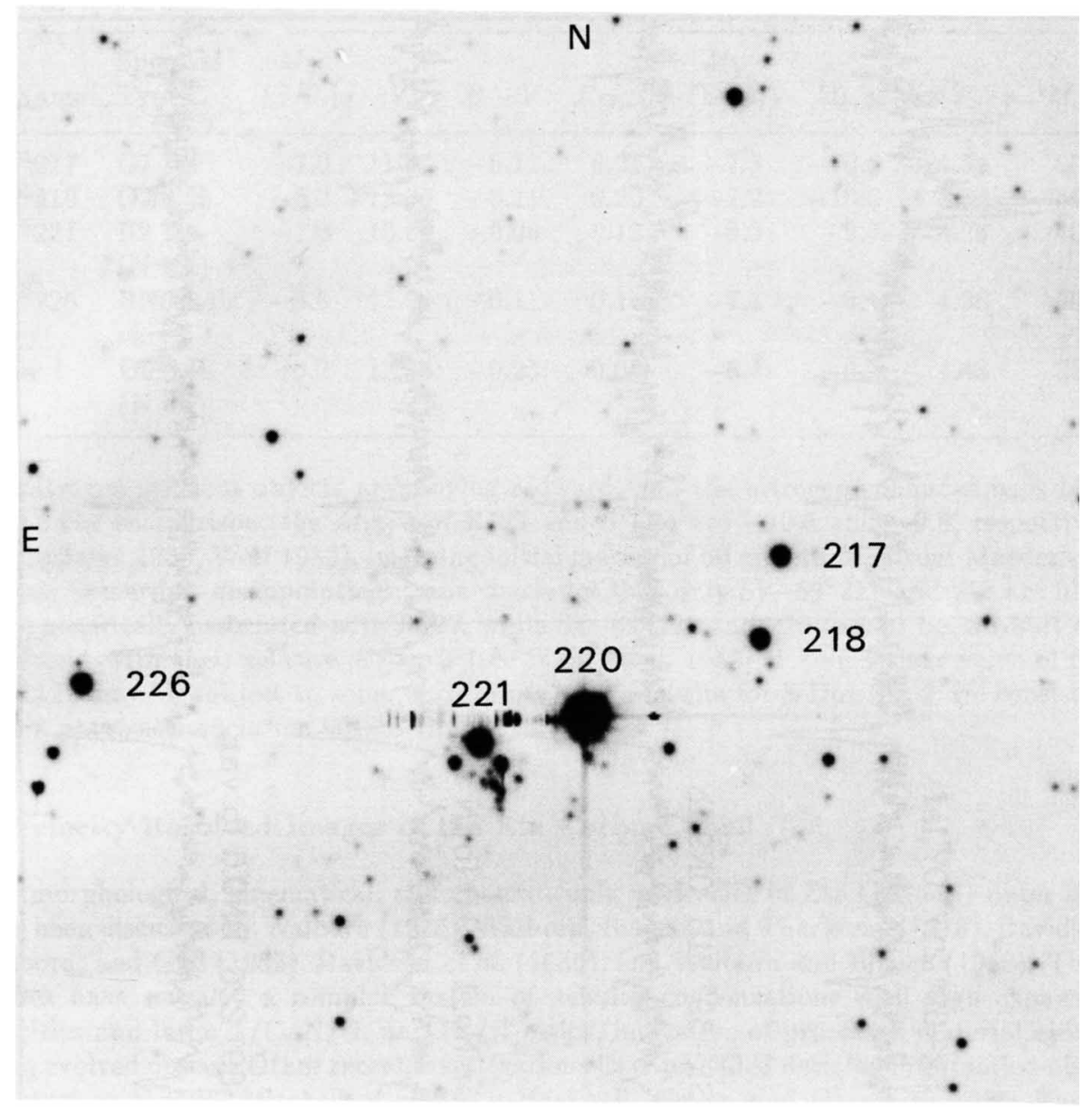

Figure 1. The field of R127 (NGC 2055). Twenty-sec R CCD frame kindly obtained by Dr. N. Suntzeff at the CTIO $0.9 \mathrm{~m}$ on Oct. 28,1988 (the overexposed image of R127 has produced some CCD artefacts). Identification numbers from the $-69^{\circ}$ zone of Sanduleak (1970) are given. $\mathrm{Sk}-69^{\circ} 217$ and 218 are separated by $9^{\prime \prime}$ in declination.

Although the uncertainties and discrepancies among current evolutionary tracks for massive stars are well known, those by Maeder (1990) are the most comprehensive and applicable to the present investigation. While further observational and theoretical work is required, there is growing evidence that the relative $\mathrm{CNO}$ abundances in $\mathrm{OB}$ supergiant spectra provide essential evolutionary diagnostics (Walborn 1988), specifically the direction of current motion in the HR diagram (redward or blueward) for a given mass range. Accordingly, the mass estimates in Table 1 have been derived with the assumption that 


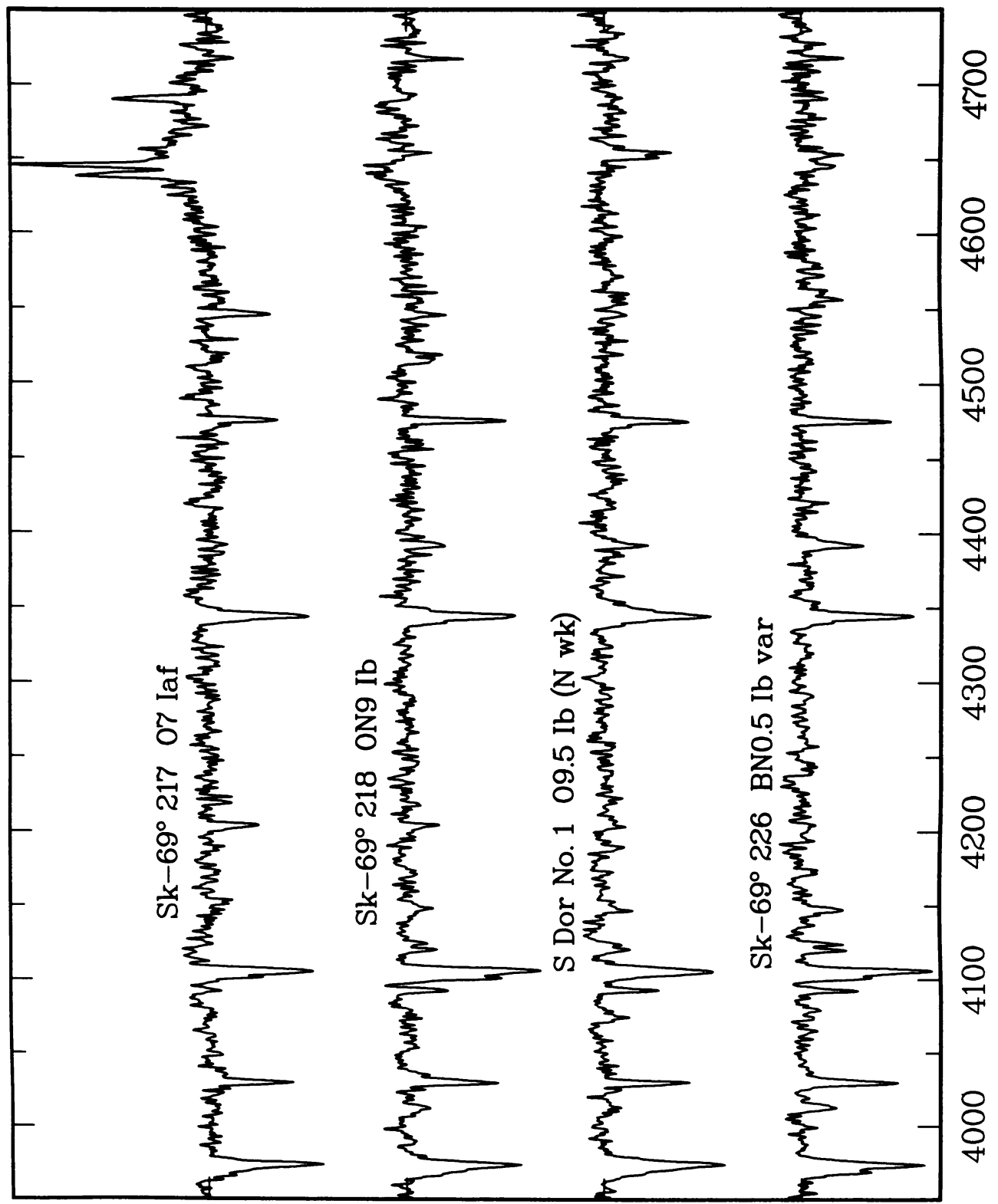

Figure 2. Blue-violet digital spectrograms of LMC OB supergiants near R127 and S Dor. Wavelengths in $\AA$ are given and the ordinate ticks are separated by 0.5 linear-intensity, continuum-flux units. See Walborn and Fitzpatrick (1990) for identification of spectral features and the text for discussion. 
TABLE 1. Parameters of OB supergiant companions to R127 and S Dor

\begin{tabular}{llcccccccc}
\hline Sk/Name & $\begin{array}{l}\text { Spectral } \\
\text { Type }\end{array}$ & $\begin{array}{c}M_{V} \\
(\mathrm{~S} . \mathrm{T} .)\end{array}$ & $V$ & $B-V$ & $E_{B-V}$ & $\begin{array}{c}M_{V} \\
(\mathrm{D} . \mathrm{M} .)\end{array}$ & $M_{\text {bol }}$ & $\log T_{\text {eff }}$ & $M_{\odot}$ \\
\hline$-69^{\circ} 217$ & O7 Iaf & -7.0 & 11.97 & -0.11 & 0.21 & -7.3 & -10.6 & 4.54 & 60 \\
$-69^{\circ} 218$ & ON9 Ib & -6.2 & 12.00 & -0.11 & 0.20 & -7.2 & -10.3 & 4.51 & 55 \\
$-69^{\circ} 221$ & B2 Ia & -7.0 & 10.63 & -0.06 & 0.12 & -8.3 & -9.7 & 4.26 & 40 \\
& (N wk) & & & & & & & & \\
$-69^{\circ} 226$ & BN0.5 Ib & -6.0 & 11.94 & -0.11 & 0.14 & -7.1 & -9.1 & 4.36 & 30 \\
& var & & & & & & & & \\
S Dor 1 & $\begin{array}{l}\text { O9.5 Ib } \\
\text { (N wk) }\end{array}$ & -6.2 & $12.35:$ & $-0.25:$ & $0.05:$ & $-6.4:$ & $-9.2:$ & 4.48 & $35:$ \\
& (N) & & & & & & & \\
\hline
\end{tabular}

the nitrogen-deficient objects are moving redward, and the nitrogen-enhanced ones blueward. For comparison, the $M_{b o l}$ 's of R127 and S Dor are -10.5 and -9.8 , respectively (Humphreys 1989, Wolf 1989), implying initial masses of 60 and $40 M_{\odot}$ from Maeder's diagram. Somewhat disappointingly, one concludes that only Sk-69 217 and 218 are likely to be generically associated with $\mathrm{R} 127$, while $\mathrm{Sk}-69^{\circ} 221$ and 226 cannot be, a result also consistent with their relative $E_{B-V}$ 's (see Stahl et al. 1983, although their value of 0.20 for R127 may be subject to some uncertainty). The results for $S$ Dor No. 1 are consistent with a physical association with S Doradus.

\section{Velocity-Resolved Images of the Eta Carinae Shell}

The morphological, kinematical, and spectroscopic intricacies of Eta Carinae's outer shell have been discussed by Walborn (1976), Walborn, Blanco, and Thackeray (1978), Davidson, Walborn, and Gull (1982), Davidson et al. (1986), and Walborn and Blanco (1988). These studies have revealed a complex system of nebular condensations with high expansion velocities and large $\mathrm{N} / \mathrm{C}, \mathrm{N} / \mathrm{O}$, and $\mathrm{He} / \mathrm{H}$ ratios, indicative of processed material ejected by an evolved object. Other recent investigations have provided detailed information about structure in the IR (Mitchell et al. 1983; Hackwell, Gehrz, and Grasdalen 1986; Russell et al. 1987) and in X-rays (Chlebowski et al. 1984), IR spectra (Allen, Jones, and Hyland 1985), very high radial velocities and polarization (Meaburn, Wolstencroft, and Walsh 1987; Dufour 1989), UV spectra (Viotti et al. 1989), and photometry from coronagraphic images (Burgarella and Paresce 1990).

As a further contribution toward disentangling the complex kinematical structure of this object, which exhibits large changes on very small angular scales, NRW and MMP obtained velocity-resolved images with the Rutgers Fabry-Perot (Schommer et al. 1988) at the CTIO $4 \mathrm{~m}$ telescope on the night of Feb. 10, 1988. The $\mathrm{H} \beta$ line was selected since it is strong and in a relatively clear spectral region throughout the object. The instrument provided a spectral resolution of $2.5 \AA(154 \mathrm{~km} / \mathrm{sec})$; the detector was a 512 -square, 15 $\mu$-pixel TI CCD. Two series of 29 images each extending from $4847.5 \AA$ to $4882.5 \AA$ (on axis) in steps of $1.25 \AA$ were obtained: one with exposure times of $60 \mathrm{sec}$ behind a central occulting wire for the fainter outer structure, and the other of $1 \mathrm{sec}$ unocculted for the inner structure. Unfortunately, the available $\mathrm{H} \beta$ pre-selecting interference filter suffered 


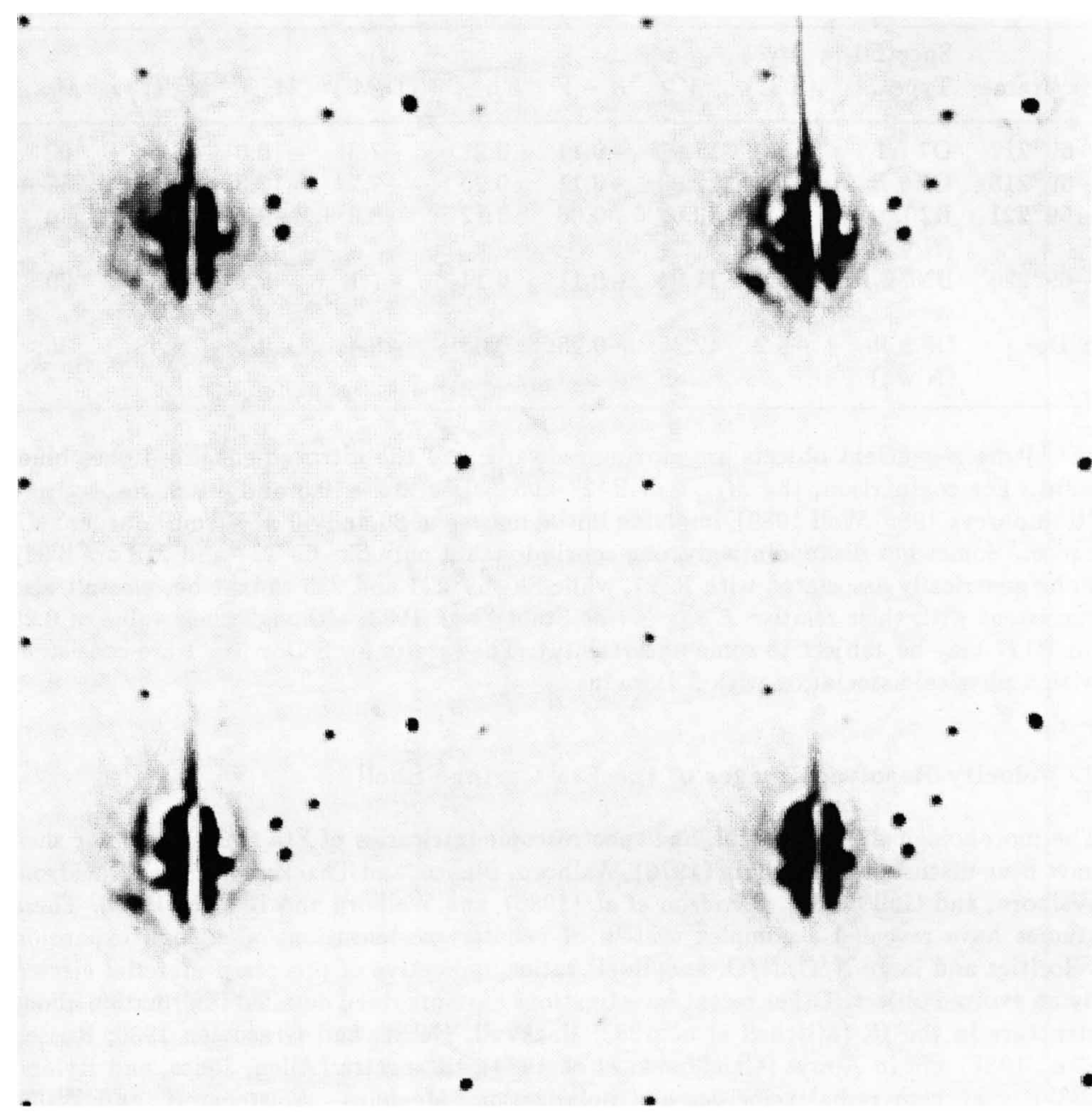

Figure 3. Velocity-resolved images of Eta Car. The heliocentric radial velocities at the object center are $-174 \mathrm{~km} / \mathrm{sec}$ in the upper left panel, $+58 \mathrm{~km} / \mathrm{sec}$ in the upper right, $+289 \mathrm{~km} / \mathrm{sec}$ at lower left, and $+521 \mathrm{~km} / \mathrm{sec}$ at lower right. The vertical spike is caused by the central occulting wire, which is in PA $130^{\circ}$. The two similar stars immediately to the right of the object are separated by $5^{\prime \prime}$.

from internal reflections and produced a triangular stellar PSF in the images. This problem has been addressed for the long-exposure series with maximum-entropy techniques by INE at the STScI (by means of software kindly made available by John Skilling), resulting in a substantially improved, symmetrical PSF with a typical FWHM of 1.2 . The structure in the outer homunculus and the outer condensations has likewise been strikingly enhanced. 
The systematic velocity behavior of several bright outer condensations shows good agreement with the spectroscopic descriptions of Davidson et al. (1982), Meaburn et al. (1987), and Dufour (1989), but many other spatial/velocity structural relationships are revealed for the first time. Four of these images are shown in Figure 3; they have not yet been corrected for the FP off-axis wavelength variation, but the velocity range across the object images is only $35 \mathrm{~km} / \mathrm{sec}$, which is hardly significant relative to the velocity resolution and displayed intervals. Further analysis of these remarkable images promises to advance qualitatively our knowledge of the Eta Carinae shell.

\section{References}

Allen, D. A., Jones, T. J., and Hyland, A. R. (1985) Ap. J., 291, 280.

Ardeberg, A., Brunet, J.-P., Maurice, E., and Prev́ot, L. (1972) Astr. Ap. Suppl., 6, 249.

Bohannan, B. and Walborn, N. R. (1989) Pub. A.S.P., 101, 520.

Burgarella, D. and Paresce, F. (1990) in preparation.

Chlebowski, T., Seward, F. D., Swank, J., and Szymkowiak, A. (1984) Ap. J., 281, 665.

Davidson, K., Dufour, R. J., Walborn, N. R., and Gull, T. R. (1986) Ap. J., 305, 867.

Davidson, K., Moffat, A. F. J., and Lamers, H. J. G. L. M. (eds.) (1989) IAU Colloq. 113, Physics of Luminous Blue Variables (Kluwer).

Davidson, K., Walborn, N. R., and Gull, T. R. (1982) Ap. J. (Letters), 254, L47.

Dufour, R. J. (1989) Rev. Mexicana Astron. Astrof., $18,87$.

Fitzpatrick, E. L. (1988) Ap. J., 335, 703. . (1990) in preparation.

Hackwell, J. A., Gehrz, R. D., and Grasdalen, G. L. (1986) Ap. J., 311, 380.

Humphreys, R. M. (1989) in IAU Colloq. 113, Physics of Luminous Blue Variables, ed. K. Davidson, A. F. J. Moffat, and H. J. G. L. M. Lamers (Kluwer), p. 3.

Humphreys, R. M. and McElroy, D. B. (1984) Ap. J., 284, 565.

Isserstedt, J. (1975) Astr. Ap. Suppl., 19, 259.

Johnson, H. L. (1966) Ann. Rev. Astr. Ap., 4, 193.

Leitherer, C., Appenzeller, I., Klare, G., Lamers, H. J. G. L. M., Stahl, O., Waters, L. B. F. M., and Wolf, B. (1985) Astr. Ap., 153, 168.

Maeder, A. (1990) Astr. Ap. Suppl., in press.

Meaburn, J., Wolstencroft, R. D., and Walsh, J. R. (1987) Astr. Ap., 181, 333.

Mitchell, R. M., Robinson, G., Hyland, A. R., and Jones, T. J. (1983) Ap. J., 271, 133.

Russell, R. W., Lynch, D. K., Hackwell, J. A., Rudy, R. J., Rossano, G. S., and Castelaz, M. W. (1987) Ap. J., $321,937$.

Sanduleak, N. (1970) Cerro Tololo Inter-Am. Obs. Contr., No. 89.

Schild, H. (1987) Astr. Ap., 173, 405.

Schommer, R. A., Caldwell, N., Wilson, A. S., Baldwin, J. A., Phillips, M. M., Williams, T. B., and Turtle, A. J. (1988) Ap. J., 324, 154.

Stahl, O., Wolf, B., Klare, G., Cassatella, A., Krautter, J., Persi, P., and Ferrari-Toniolo, M. (1983) Astr. Ap., 127, 49.

Viotti, R., Rossi, L., Cassatella, A., Altamore, A., and Baratta, G. B. (1989) Ap. J. Suppl., 71, 983.

Walborn, N. R. (1972) A. J., 77, 312. 
. (1973) A. J., 78, 1067.

(1976) Ap. J. (Letters), 204, L17.

(1977) Ap. J., 215, 53.

(1983) Ap. J., 265, 716.

(1988) in IAU Colloq. 108, Atmospheric Diagnostics of Stellar Evolu-

tion, ed. K. Nomoto (Springer-Verlag), p. 70.

(1989) in IAU Colloq. 113, Physics of Luminous Blue Variables, ed. K.

Davidson, A. F. J. Moffat, and H. J. G. L. M. Lamers (Kluwer), p. 27.

Walborn, N. R. and Blanco, B. M. (1988) Pub. A.S.P., 100, 797.

Walborn, N. R., Blanco, B. M., and Thackeray, A. D. (1978) Ap. J., 219, 498.

Walborn, N. R. and Fitzpatrick, E. L. (1990) Pub. A.S.P., 102, 379.

Wolf, B. (1989) in IAU Colloq. 113, Physics of Luminous Blue Variables, ed. K. Davidson,

A. F. J. Moffat, and H. J. G. L. M. Lamers (Kluwer), p. 91.

\section{DISCUSSION}

Montmerle: A question about "coevality". Why do you rule out some stars as part of the R127 group on the basis of their masses (hence lifetimes)? In a given association, stars are not necessarily born at the same time: the differences in ages may be larger than the lifetimes of the stars themselves.

Walborn: At least in rich OB clusters one can distinguish Orion Nebula, Carina Nebula, Scorpius OB1, and $\mathrm{h} / \chi$ Persei phases, in order of increasing ages from $\lesssim 10^{6}$ to $\lesssim 10^{9}$ years. Therefore, the spread in formation times within a given object must be less than the age differences among these phases, i.e., a couple of million years. Of course, in large associations there can be sequential formation of different subgroups. My purpose was to find objects coeval with the LBV's.

Smith, Lindsey: I noticed in the spectra of the Large Cloud supergiants that you showed, that the nitrogen emission lines were significantly brighter than HeII4686. Is that unusual, is it significant, and do you know what controls these relative brightnesses?

Walborn: There is a range of strengths both in the Galaxy and in the LMC. There must be a range of envelope densities. e.g. within the association Sco OB1. In the Galaxy one has HD 151804 and HD 152408 right next to each other.

Underhill: Because the relative populations of the levels between which CIII4650 and NIII 4096,4103 occur are very sensitive to NLTE effects, due chiefly to dielectronic recombination, you should be cautious about interpreting changes in the apparent strength of these lines to abundance differences.

Walborn: Of course, a definitive quantitative analysis is required to derive abundances. However, if I see three spectra of identical temperature/luminosity classes, one with both $C$ and $N$ strong, another with $C>N$, and the other with $N>C$, my intuition suggests abundance anomalies as the most reasonable hyothesis. 